\title{
Distribution of receptor sites on large glycoprotein molecules by electron microscopy
}

\author{
Application to epiglycanin
}

\author{
Henry S. SLAYTER and John F. CODINGTON \\ Sidney Farber Cancer Institute, and the Department of Physiology, Harvard Medical School, \\ Boston, MA 02115, and the Laboratory for Carbohydrate Research, Massachusetts General Hospital, and \\ Department of Biological Chemistry, Harvard Medical School, Boston, MA 02114, U.S.A.
}

(Received 30 May 1980/Accepted 10 September 1980)

\begin{abstract}
Electron microscopy was used to map the loci of immunochemically active sites on individual glycoprotein molecules. The positions of specific galactose residues and asparagine-linked carbohydrate chains containing specific mannose residues in epiglycanin, a glycoprotein of extended conformation from the surface of TA3 mouse mammary tumour cells, were observed in complexes with Ricinus communis toxin and concanavalin A respectively. The maximum number of Ricinus communis toxin molecules attached to a single epiglycanin molecule was 23 , and the average number was 16. Only one concanavalin A molecule was observed attached to any epiglycanin molecule, and this at one end of the molecule, suggesting the presence of only one receptor for this lectin. By means of this new approach for mapping specific residues, evidence has been obtained that suggests microheterogeneity in epiglycanin with respect to the locations of carbohydrate chains containing receptors for Ricinus communis toxin.
\end{abstract}

A method for the rapid determination of the locations of specific sites, whether carbohydrate or protein, along the polypeptide chain in large glycoproteins of extended conformation would be important in studies of structure-function relationships, particularly in the case of glycoproteins at the surfaces of mammalian cells. Information regarding the position of the receptor for a hormone, a virus or an antibody may be of potential biomedical importance. Yet, very little is known regarding the location of specific carbohydrate chains in cell-surface glycoproteins. Some exceptions are the glycoprotein of the human erythrocyte that contains the $\mathbf{M}$ and $\mathbf{N}$ blood group specificities (Tomita \& Marchesi, 1975; Lisowska \& Wasniowska, 1978), and certain histocompatibility antigens (Parham et al., 1977; Barnstable et al., 1978). With these substances the location of carbohydrate chains was determined in the course of elucidating complete amino acid sequences, a procedure that does not provide information regarding specific receptor activity. The elucidation of the positions of exposed amino acid residues in a non-membrane glycoprotein, $\alpha-1$ acid glycoprotein, was achieved by Schmid et al. (1976)

Abbreviation used: SDS, sodium dodecyl sulphate. using chemical methods. In this paper we describe a method for the visualization by electron microscopy of the locations of receptors in large glycoproteins of extended conformation.

The method described here involves the complexing of a group-specific macromolecule, such as an antibody or a lectin, with an asymmetric glycoprotein, followed by the direct visualization of the complex in the electron microscope. Investigations of glycoprotein structure illustrate the scope of electron microscopic replica methods for the visualization of macromolecular specimens (Slayter, 1976; Slayter \& Codington, 1973; Slayter et al., 1974; Slayter \& Coligan, 1975, 1976).

This method may be uniquely applicable to the investigation of a problem for which no solution has yet been possible; namely, the relationship between microheterogeneity and biological activity in glycoproteins. Since heterogeneity involving the presence of 'incomplete' carbohydrate chains appears to be an almost universal characteristic of this type of macromolecule (Spiro, 1973), information regarding the type of variation occurring at a particular site could add a new dimension to our understanding of the biological functions of glycoproteins. However, it is not yet known whether or not microheterogeneity 
is related to the proper biological functioning of glycoproteins.

As a model for this investigation we have utilized an endogenous cell-surface glycoprotein of the TA3-Ha mammary carcinoma ascites tumour of the strain A mouse. The molecular configuration and apparent shape of this long extended rod-like molecule as studied by physicochemical (Slayter \& Codington, 1973; Codington et al., 1975b; Codington et al., 1979; Van den Eijnden et al., 1979) and immunochemical (Codington et al., 1975a,b; Matsumoto et al., 1980) approaches, suggests it as a suitable model for this type of study, in particular since epiglycanin has already been studied extensively by electron microscopic methodology (Slayter \& Codington, 1973; Codington et al., 1979).

Use of electron microscopy of individual molecules complexed with specific proteins has made it possible to determine the positions of active carbohydrate sites along the polypeptide chain of epiglycanin, as well as to elucidate both similarities and differences in the distribution of carbohydrate chains among individual molecules occurring in a particular fraction. In addition, we have been able to obtain data regarding the number and spacing of two types of carbohydrate chains in this glycoprotein.

\section{Materials and methods}

\section{Epiglycanin}

Epiglycanin was isolated from the ascites fluid of A/HeJ mice bearing TA3-Ha ascites cells and was purified by gel filtration, by procedures previously described (Codington et al., 1979).

\section{Lectins}

Ricinus communis toxin was purified by the method of Olsnes et al. (Olsnes \& Pihl, 1973; Olsnes et al., 1974) and gave a single band with SDS/ polyacrylamide-gel electrophoresis and isoelectric focusing. Concanavalin A was obtained from Miles Laboratories, Elkhart, IN, U.S.A.

\section{Complexing procedures}

In a typical experiment $20 \mu \mathrm{g}$ of epiglycanin was reacted for $90 \mathrm{~min}$ in $50 \mu \mathrm{l}$ of $0.1 \mathrm{M}$-ammonium acetate, pH6.5, with an amount of Ricinus communis toxin equal to 200 molecules of lectin per molecule of epiglycanin. The solution was then eluted with the same solvent from a column of Bio-Gel P-300 $(0.6 \mathrm{~cm} \times 4 \mathrm{~cm})$. Several fractions collected after the exclusion volume $\left(V_{0}\right)$ of the column were prepared for electron microscopy.

In complexing epiglycanin and concanavalin $\mathrm{A}$, six $100 \mu \mathrm{l}$ portions of affinity-purified* epiglycanin $(150 \mu \mathrm{g} / \mathrm{ml})$ were added with stirring over $20 \mathrm{~min}$ at $0^{\circ} \mathrm{C}$ to $100 \mu \mathrm{g}$ of concanavalin $\mathrm{A}$ in $50 \mu \mathrm{l}$ of $0.10 \mathrm{M}$-sodium acetate ( $\mathrm{pH} 6.5$ ) containing $\mathrm{Ca}^{2+}$, $\mathrm{Mg}^{2+}$ and $\mathrm{Mn}^{2+}(1.0 \mathrm{mM})$. After $5 \mathrm{~h}$ at $0-4^{\circ} \mathrm{C}$ the solution was dialysed against ammonium acetate $(0.15 \mathrm{M}, \mathrm{pH} 6.5)$, concentrated to $300 \mu \mathrm{l}$, and passed through a calibrated column of Bio-Gel P-300 (eluent, $0.15 \mathrm{M}$-ammonium acetate, $\mathrm{pH} 6.5$ ) to remove unbound lectin. The column fractions were then prepared for electron microscopy.

\section{Electron microscopy}

Procedures used for electron microscopy have been presented in detail elsewhere (Slayter, 1976; Slayter, 1978). Briefly, complexes in $0.15 \mathrm{M}$ ammonium acetate, pH6.5, were applied as an aerosol to freshly cleaved mica and then rotary shadow-cast with platinum. Carbon-backed replicas were then stripped from the mica substrate and photographed at $80 \mathrm{kV}$ and magnification $25000 \times$.

\section{Results}

\section{Receptors for Ricinus communis toxin}

In Plate 1 complexes formed from the interaction of epiglycanin and Ricinus communis toxin (Plate $1 b)$ are shown along with uncomplexed epiglycanin (Plate 1a). Images of complexes were scored to scale on graph paper with regard to number and position (percentage of total length from the left side in Fig. 1) of attached lectin molecules. The resulting maps of 22 individual complexes were cut out and matched in groups for best fit. Polarity was determined by pattern matching, since neither end-group has yet been identified in electron micrographst. When a frequency histogram for all of these complexes was plotted (Fig. 1), it was found that there are about 10-12 positions on epiglycanin molecules where the lectin frequently binds. Ten sites are identified in Fig. 1. These are in the vicinity of 1 , $3,17,21,34,50,65,74,85$ and $100 \%$ of epiglycanin length. Because of the extra density of marker population at the left end of the histogram in Fig. 1, it seems likely that unresolved sites are contributing to the pattern in regions $1-5$ and $15-23 \%$. The

* Epiglycanin was adsorbed on a column of concanavalin A-Sepharose 4B, and the fraction that was specifically eluted with methyl $\alpha$-D-mannopyranoside was used.

† This approach has hitherto been hindered by our inability to distinguish in the electron micrographs between the amino and carboxy terminals in epiglycanin. In practice, complex matching was based upon apparent polarization revealed by the end of molecules bearing the largest number of attached lectin particles, on the assumption that the asymmetry of individual molecules can be used for polarization in the matching process. At present, we have no means of polarizing binding patterns of different markers such as the ones used in this study. 

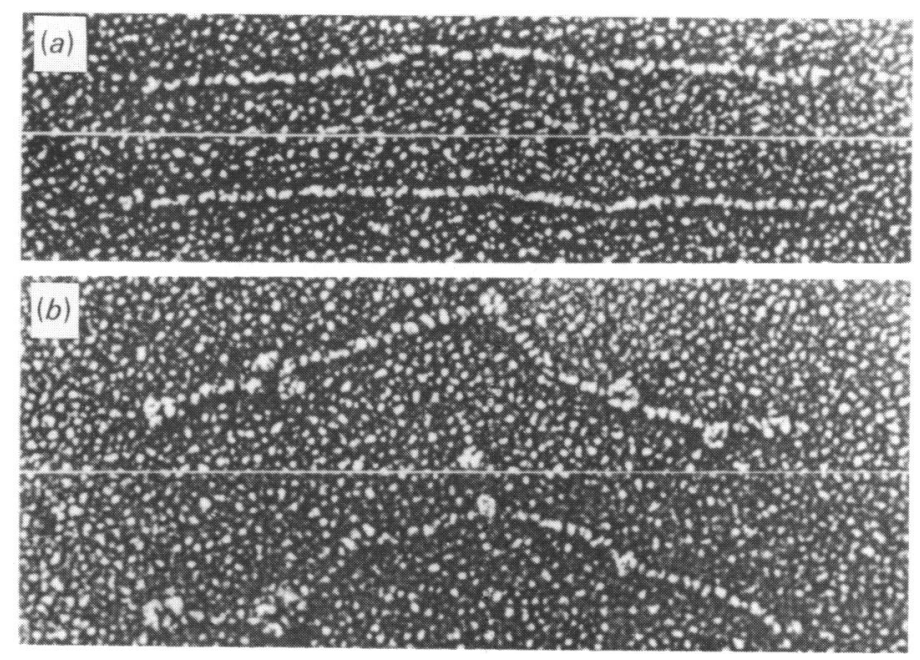

EXPLANATION OF PLATE 1

Electron micrographs of epiglycanin

(a) Epiglycanin molecules obtained from ascites fluid essentially intact. Rotary shadow cast with platinum. Magnification 120000x. (b) Epiglycanin complexed with Rieinus communis toxin. Magnification 130000x. 


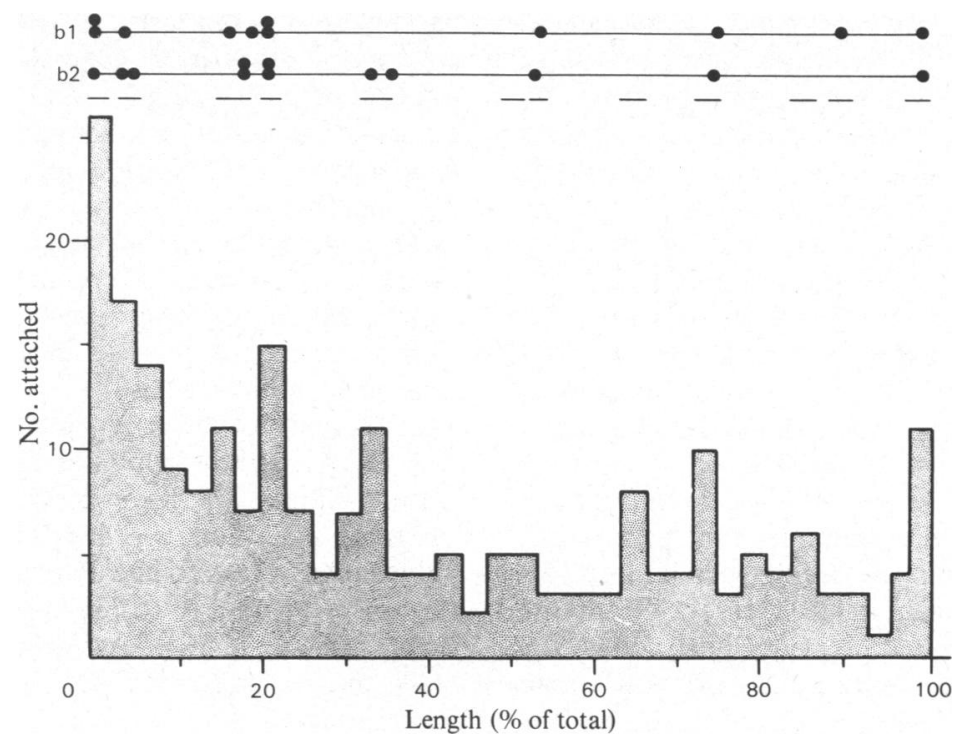

Fig. 1. Histogram plot of the frequency distribution of Ricinus communis bound to epiglycanin Number versus percentage of the total length of epiglycanin is shown. Regions where Ricinus binds epiglycanin are designated by bars. Distribution patterns of two nearly identical complexes (b1, b2) are plotted above the Figure.

resolution of the method for determining Ricinus sites is thus at best 3-5\% of epiglycanin length or $15-25 \mathrm{~nm}$.

Strikingly similar patterns were observed in small numbers of complexes. The probability of finding two essentially identical patterns, as illustrated by the two complexes in Plate $1(b)$, were calculated by a multinomial test. The probability of placing the 12 nodes associated with the upper complex on the lower complex was calculated with 20 equal subintervals of the epiglycanin length. The chance of obtaining a second pattern at least as similar to the first, as was found, was calculated to be 1 in $10^{7}$ under a chance-allocation mechanism (out of 22 complexes). On the other hand, the majority of complexes examined showed differences in binding patterns.

\section{Concanavalin $A$ binding sites}

Complexes of concanavalin A affinity-purified epiglycanin with concanavalin A lectin when visualized by electron microscopy appeared rather uniformly to possess a single lectin-sized particle very close to one end of epiglycanin. Concanavalin A appeared to bind to about half of the epiglycanin molecules. Quantitatively, a greater proportion of affinity-purified* epiglycanin molecules appeared to bind concanavalin A than did epiglycanin that had not been affinity-purified. Plate 2 shows electron micrographs of a field of epiglycanin-concanavalin A complexes and of individual complexes at a high magnification comparable with that of Plate 1.

\section{Discussion}

Experiments presented here provide information concerning the location, within epiglycanin molecules, of two types of binding site: the binding site for Ricinus communis toxin and the binding site for concanavalin A lectin.

Chemical data indicate that epiglycanin of mol.wt. 500000 possesses approx. $520 O$-glycosyl-linked carbohydrate chains (Codington et al., 1975b; Van den Eijnden et al., 1979). In an investigation by Van den Eijnden et al. (1979) five different types of reduced oligosaccharides, each representing a different type of $\boldsymbol{O}$-glycosyl-linked chain, were removed from epiglycanin by chemical methods, purified and characterized. Receptor sites for most of the large number of lectins used in the elucidation of the chemical structure of epiglycanin are present mainly in these five chains. For example, the receptor for Ricinus communis agglutinin and toxin is probably part of a tetrasaccharide and a hexasaccharide, but is masked by a sialic acid residue in the latter. On the basis of haemagglutination-inhibition studies with epiglycanin fractions, it was suggested that the receptor for Ricinus communis agglutinin in epiglycanin was a $\beta$-D-Gal $(1 \rightarrow 4)-\beta$-D-GlcNAc sequence. Given the proportion of this disaccharide present as a terminal sequence residue in a reduced tetrasaccharide isolated from epiglycanin after alkaline borohydride reduction (Codington et al., 1975b; Van den Eijnden et al., 1979), it may be calculated that there are 20-25 such receptors in epiglycanin. 
The presence of Ricinus communis receptors was demonstrated by the significant inhibitory activity of both epiglycanin and neuraminidase-treated epiglycanin against the agglutination by Ricinus communis agglutinin of TA3-Ha cells, TA3-St cells and human erythrocytes (Codington et al., 1975b; Matsumoto et al., 1980). The subline of the TA3 tumour, which possesses epiglycanin (TA3-Ha), was found to possess $1.1 \times 10^{7}$ more receptors for Ricinus communis agglutinin than the TA3-St subline, which contains no epiglycanin (Matsumoto et al., 1980). Binding of Ricinus communis agglutinin to the TA3-Ha cells was specifically inhibited by lactose (Matsumoto et al., 1980). In the present experiments, the maximum number of lectin molecules found to attach to single epiglycanin molecules was 23 , and the mean value was 16 . From the observed distribution it is concluded that the carbohydrate chains bearing the Ricinus toxin receptor are fairly evenly distributed along the $500 \mathrm{~nm}$ long epiglycanin molecule, although they are somewhat more concentrated towards one end. Comparison of the summed distributions of all patterns reveals good correspondence with the expected number of binding sites.

One reason why fewer than the theoretical number of sites might be found is the possibility of two sites close together, which given the estimated present resolution of the method $(15-25 \mathrm{~nm})$ may give rise to a wide peak, representing two discrete sites. Thus 20-25 sites might give rise to 10-12 apparent loci of larger than expected size. However, the possibility for considerable variations of peptide-bond length in the case of epiglycanin may well add to the uncertainty as to the precise positions.

Some strikingly similar complexes were obtained, indicating either that some sites are preserved at higher frequency than others or that some fraction of the epiglycanin material is in fact homogeneous. Failure of all complexes examined to match perfectly may, however, be considered as evidence that epiglycanin is microheterogeneous with respect to loci of Ricinus binding sites. The occurrence of such microheterogeneity may be readily explained on the basis of a certain amount of randomness in the glycosylation process. Such an explanation seems plausible for epiglycanin, since no inconsistency has yet been observed in the hypothesis that the various $O$-glycosyl-linked chain types in epiglycanin represent discrete stages in the completion of a single biosynthetic sequence of events, which culminates in the formation of the hexasaccharide (Van den Eijnden et al., 1979). It should be noted, however, that the course of glycosylation in epiglycanin may be strongly influenced by the amino acid sequence, which is not yet known. Assuming that the epiglycanin molecules investigated in this study possess a common amino acid sequence, such variations might conceivably arise as a result of variability in the site of cleavage of epiglycanin by proteinases in the process of shedding from the cell. Since little is known about the origin, biosynthesis or structure of endogenous intrinsic tumour-associated cell-surface glycoproteins such as epiglycanin, however, the possibility must be considered that epiglycanin is a mixture of related glycoproteins in which minor variations in the amino acid sequence may cocur. There are yet other possibilities that may account for the observed variations.

Flexibility of the polypeptide chain may result in localized length reduction, conceivably in more than one location (we have previously found that the average extension per amino acid residue in epiglycanin is close to the Pauling \& Corey figure of $0.36 \mathrm{~nm}$; Pauling \& Corey, 1951); in the complexed molecule the average extension per residue may be less, due to alterations in charge distribution; some non-specific or anomalous attachment of lectin to epiglycanin must be considered.

A consistent small percentage $(0.3-0.4 \%)$ of mannose is always detected in epiglycanin by gas chromatography. This would represent 9-12 mannose residues per molecule and suggests the possible presence of one or two asparagine-linked ( $N$-chains) per molecule. Thus there would be one or possibly two mannose-specific sites available per epiglycanin of mol.wt. about 500000 . Further evidence for concanavalin A receptors in epiglycanin was obtained by inhibition studies. Epiglycanin demonstrated significant inhibition of the agglutination of TA3-St cells and rabbit erythrocytes by concanavalin $\mathbf{A}$ (Matsumoto et al., 1980).

Evidence is presented here to support the existence in approx. half of the epiglycanin molecules studied of at least one carbohydrate chain containing a receptor for concanavalin $\mathrm{A}$. In addition to visual evidence from electron micrographs, a large proportion ( $\simeq 55 \%)$ of epiglycanin was found to bind to concanavalin A-Sepharose affinity columns and to be specifically eluted with methyl $\alpha$-D-mannopyranoside. The total recovery of epiglycanin from the column was $>75 \%$, based on sialic acid determination (Warren, 1959), after its cleavage from the glycoprotein with neuraminidase. In studies by Narasimhan et al. (1979) and Baenziger \& Kiete (1979), concanavalin A receptors are found in asparagine-linked carbohydrate chains of both the 'high mannose' and 'complex' types. All 'complex' type oligosaccharides do not bind, however, as the presence of certain residues may decrease or eliminate binding activity.

Our results indicate that at least one concanavalin $\mathbf{A}$ receptor is present at, or very near, one end of the molecule. Of several hundred molecules examined no epiglycanin molecule appeared to possess more than one bound lectin particle, 
although, as mentioned above, many molecules possessed no complexed lectin. Two plausible explanations for this apparent heterogeneity are suggested. (1) Some molecules possess no asparagine $(N$-glycosyl)-linked chain. This possibility seems improbable. (2) $\mathrm{N}$-Glycosyl-linked chains are present in all molecules, but some contain no concanavalin A receptor. Thus there appears to be no heterogeneity with regard to the locus of the concanavalin A receptor when it is present, but there may be variations in the structure of the chain itself.

We have observed that in complexes of epiglycanin with Ricinus communis toxin some sites contain more than one lectin despite removal of excess lectin from reaction mixtures to prevent non-specific association. The explanation for this observation is uncertain. We suggest, however, that non-specific interactions may occur either between two or more protein markers or between the protein markers and epiglycanin. Some lectins were found to dissociate from the glycoprotein in the dilute solutions required during preparation of specimens for electron microscopy, indicating that limited binding energies are a problem in exploiting the carbohydrate-protein interactions used here. Thus some dissociation might occur at the high saltconcentrations produced during the rapid dehydration of ammonium acetate solutions of complexes during preparation for electron microscopy. This could result in some non-specific reassociation of lectin with the complexes. However, the phenomenon was not observed in the case of complexes between epiglycanin and concanavalin $A$.

Use of additional specific lectins and antibodies to specific haptenic structures should eventually make it possible to pinpoint a number of different types of receptor loci, not only within epiglycanin molecules, but on other important cell-surface glycoproteins. At present, the results reported here may be less important than the methodology that they illustrate. It is the intent of this report to present a rapid and economic method for determining the distribution of immunochemically specific sites in extended glycoprotein molecules. Whereas classical immunology deals with the semiquantitative study of the precipitation reactions of large molecules (such as epiglycanin), this electron microscopic approach permits the study of details of the structure of individual precipitating macromolecular complexes.

We acknowledge the assistance of Joseph Rothschild, Joseph Potz, Cyla Silber, Pamela Walter and Helen Brinkerhoff in this work. We thank Dr. David Schlesinger of Massachusetts General Hospital for an amino acid analysis of epiglycanin. We thank Professor R. W.
Jeanloz for support and encouragement, and Dr. Phillip Lavin for statistical calculations. This work was supported by Research Grants GM 14237 from the National Institute of General Medical Sciences, FR 05526 from the Division of Research Facilities and Resources, National Institutes of Health, and by Research Grant CA 08418 and CA 18600 from the National Cancer Institute, National Institutes of Health, United States Public Health Service.

\section{References}

Baenziger, J. U. \& Kiete, D. (1979) J. Biol. Chem. 254, 2400-2407

Barnstable, C. J., Jones, E. A. \& Crumpton, M. J. (1978) Br. Med. Bull. 34, 241-246

Codington, J. F., Cooper, A. G., Brown, M. C. \& Jeanloz, R. W. (1975a) Biochemistry 14, 855-859

Codington, J. F., Linsley, K., Jeanloz, R. W., Irimura, T. \& Osawa, T. (1975b) Carbohydr. Res. 40, 171-182

Codington, J. F., Cooper, A. G., Miller, D. K., Slayter, H. S., Brown, M. C., Silber, C. \& Jeanloz, R. W. (1979) J. Natl. Cancer Inst. 63, 153-161

Lisowska, E. \& Wasniowska, K. (1978) Eur. J. Biochem. 88, 247-252

Matsumoto, I., Codington, J. F., Jahnke, M. R., Jeanloz, R. W. \& Osawa, T. (1980) Carbohydr. Res. 80, 179-189

Narasimhan, S., Wilson, J. R., Martin, E. \& Schachter, H. (1979) Can. J. Biochem. 59, 83

Olsnes, S. \& Pihl, A. (1973) Biochemistry 12, 3121-3126

Olsnes, S., Saltvedt, E. \& Pihl, A. (1974) J. Biol. Chem. 249, 803-810

Parham, P., Alpert, B. N., Orr, H. T. \& Strominger, J. L. (1977) J. Biol. Chem. 252, 7555-7567

Pauling, L. \& Corey, R. B. (1951) Proc. Natl. Acad. Sci. U.S.A 37, 241-250

Schmid, K., Chen, L. H., Occhino, J. C., Foster, J. A. \& Sperandio, K. (1976) Biochemistry 15, 2245-2254

Slayter, H. S. (1976) Ultramicroscopy 1, 341-357

Slayter, H. S. (1978) in Principles and Techniques of Electron Microscopy: Biological Applications (Hayat, M. A., ed.), vol. 9, pp. 175-245, Van Nostrand Reinhold, New York

Slayter, H. S. \& Codington, J. F. (1973) J. Biol. Chem. 248, 3405-3410

Slayter, H. S. \& Coligan, J. R. (1975) Biochemistry 14, 2323-2330

Slayter, H. S. \& Coligan, J. R. (1976) Cancer Res. 36, 1696-1704

Slayter, H. S., Cooper, A. G. \& Brown, M. C. (1974) Biochemistry 13, 3365-3371

Spiro, R. G. (1973) Adv. Protein Chem. 27, 349-467

Tomita, M. \& Marchesi, V. T. (1975) Proc. Natl. Acad. Sci. U.S.A 72, 2964-2968

Van den Eijnden, D. H., Evans, N. A., Codington, J. F., Reinhold, V., Silber, C. \& Jeanloz, R. W. (1979) $J$. Biol. Chem. 254, 12153-12159

Warren, L. (1959) J. Biol. Chem. 234, 1971-1975 